\title{
Bilateral putaminal necrosis in a comatose patient with metabolic acidosis
}

\author{
Sudhir Kumar, Chenna Rajesh Reddy, Subhashini Prabhakar
}

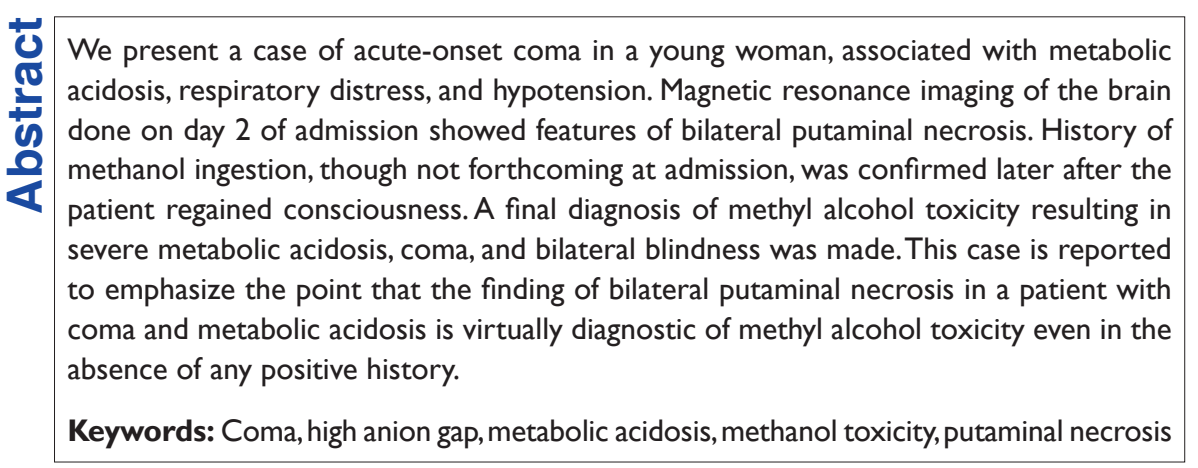

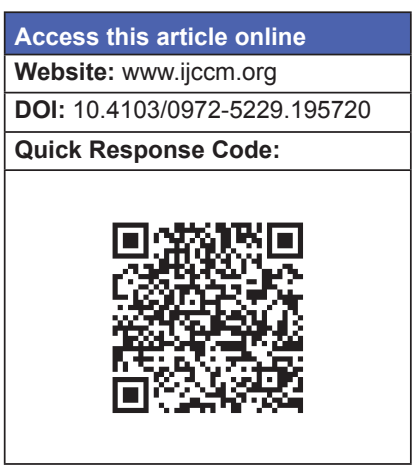

\section{Introduction}

Coma is commonly encountered in neurology practice. There are multiple etiologies of coma including metabolic and neurological. This makes it challenging to arrive at the appropriate underlying diagnosis, especially in the absence of any specific history. We recently encountered a case of coma in a young woman, with no specific leading history. The presence of severe metabolic acidosis and typical findings on brain imaging gave vital clues toward arriving at a final diagnosis.

\section{Case Report}

A 29-year-old female was brought to the emergency room in an unconscious state of about 3-h duration. She was apparently asymptomatic when seen last. There was no specific history pointing toward any likely cause of unconsciousness. On examination, she was deeply comatose. Glasgow coma scale score was 3. Pupils were $3 \mathrm{~mm}$, equal and reacting to light. Oculocephalic reflexes were present. Pulse rate was $92 / \mathrm{min}$ and blood

From:

Department of Neurology, Institute of Neurological Sciences, Apollo Hospitals, Hyderabad, Telangana, India

\section{Correspondence:}

Dr. Sudhir Kumar, Apollo Hospitals, Jubilee Hills,

Hyderabad - 500 033, Telangana, India.

E-mail: drsudhirkumar@yahoo.com pressure was $70 \mathrm{mmHg}$ systolic. She was in respiratory distress. The patient was intubated and mechanically ventilated. Dopamine was started. Hematological tests were normal. Biochemical investigations showed elevated serum creatine phosphokinase (CPK) (1280 $\mathrm{U} / \mathrm{L}$ ) and uric acid (8.7 $\mathrm{mg} \%)$. Arterial blood gasses showed severe metabolic acidosis ( $\mathrm{pH} 7.1$ ), with high anion gap $(30 \mathrm{mmol} / \mathrm{L})$. A brain magnetic resonance imaging (MRI) was obtained on day 2 of admission after hemodynamically stabilizing her. T2-weighted [Figure 1] and fluid-attenuated inversion recovery [Figure 2] images showed bilateral symmetrical hyperintense lesions involving lentiform nucleus. Mild perilesional edema was also noted.

Supportive treatment was continued, and she became conscious by day 3 and was weaned off the ventilator on day 7. She was noted to have complete blindness. She

This is an open access article distributed under the terms of the Creative Commons Attribution-NonCommercial-ShareAlike 3.0 License, which allows others to remix, tweak, and build upon the work non-commercially, as long as the author is credited and the new creations are licensed under the identical terms.

\section{For reprints contact: reprints@ @medknow.com}

How to cite this article: Kumar S, Reddy CR, Prabhakar S. Bilateral putaminal necrosis in a comatose patient with metabolic acidosis. Indian J Crit Care Med 2016;20:745-8. 


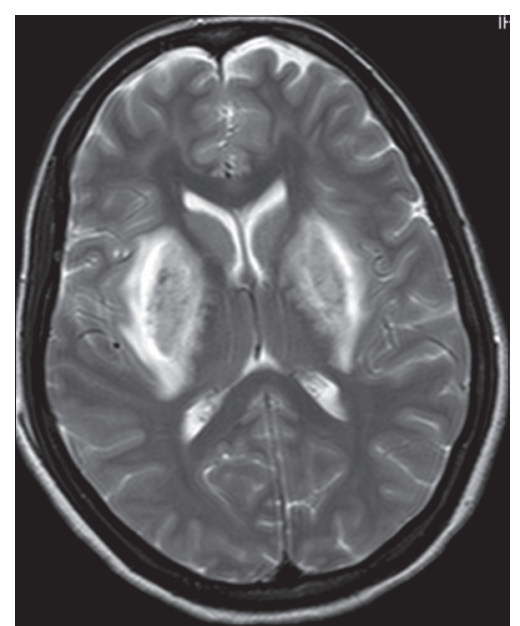

Figure I: Axial fast spin-echo T2-weighted magnetic resonance showing subcortical white matter and basal ganglia hyperintensity and low-signal intensity of bilateral putaminal foci

had dilated, nonreactive pupils. Fundus examination showed moderate papilledema and hyperemia of discs. As the clinical and radiological picture was suggestive of methyl alcohol toxicity, she was queried along those lines. The patient admitted to having consumed about $30 \mathrm{ml}$ of methyl alcohol in a suicide attempt. She was discharged 20 days after admission. At discharge, she was conscious and alert. Vision had improved to perception of hand movements, and she had no other focal neurological deficits.

\section{Discussion}

Coma has multiple causes; therefore, it is difficult to make a presumptive diagnosis in many cases. However, early etiological diagnosis is vital in starting appropriate therapy.$^{[1]}$ In patients with methanol toxicity presenting with coma, history of methanol ingestion may not be forthcoming, as happened in our patient, thereby delaying the initiation of specific treatment. However, it is important to make an early diagnosis as methyl alcohol toxicity is eminently treatable. The diagnosis of methanol toxicity can be established on the basis of following features: (i) A history of recent ingestion of illicit spirits is available and serum methanol is higher than $6.2 \mathrm{mmol} / \mathrm{L}$ $(20 \mathrm{mg} / \mathrm{dL})$ or (ii) there is a history/clinical suspicion of methanol poisoning, and serum methanol is above the limit of detection with at least two of the following: $\mathrm{pH}<7.3$, serum bicarbonate $<20 \mathrm{mmol} / \mathrm{L}$, and anion gap $\geq 20 \mathrm{mmol} / \mathrm{L}^{[2]}$

Clinical features of methanol toxicity include central nervous system symptoms (69\%), gastrointestinal complaints $(87 \%)$, visual disturbances $(69 \%)$, and metabolic acidosis (94\%). ${ }^{[3]}$ Methanol toxicity results in various neurological complications including coma,

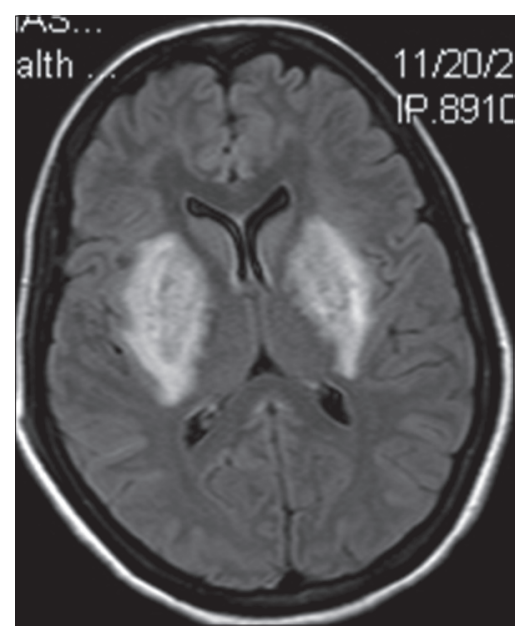

Figure 2: Axial fluid-attenuated inversion recovery image showing similar findings as Figure I

seizures, blindness, ${ }^{[4]}$ Parkinsonian syndrome,,$^{[5]}$ and intracranial hemorrhage. ${ }^{[6]}$ In our patient, pupils were equal in size and reacting normally to light at admission. Subsequent examination showed dilated, nonreactive pupils with blindness, which was suggestive of bilateral optic nerve damage. Features of optic nerve damage may be absent at admission, and it may take several hours or a few days for this to manifest.

Formic acid, the toxic metabolite of methanol, is supposed to be responsible for ocular toxicity. Generally, blindness due to methanol toxicity does not improve with time. However, a recent case of successful return to normal vision was demonstrated with intrathecal and retrobulbar administration of autologous bone marrow-derived stem cells. ${ }^{[7]}$ Vision started to improve within 3 days, and it returned to normal $(20 / 20)$ by $3^{\text {rd }}$ week. Another case demonstrated partial recovery of vision with steroid treatment (injection methylprednisolone followed by oral steroids). ${ }^{\left[{ }^{[]}\right.}$

Metabolic acidosis occurs in almost all cases of methanol toxicity. Typically, the acidosis is associated with increased anion and osmolar gaps. ${ }^{[9]}$ Confounders to the above rule are low serum methanol concentration and concomitant ethanol ingestion. Other conditions that can present with metabolic acidosis with high anion gap include intoxications with ethylene glycol, diethylene glycol, propylene glycol, and alcoholic ketoacidosis. ${ }^{[10]}$ Neuroleptic malignant syndrome (NMS) is the other condition that should be excluded. NMS can cause neurological dysfunction and metabolic acidosis. Additional clinical features in NMS include muscular rigidity, hyperthermia, and diaphoresis. Serum CPK levels are usually elevated in NMS. Other conditions which should be considered 
in the differential diagnosis of methanol toxicity are central nervous system infections (viral encephalitis, bacterial meningitis), carbon monoxide poisoning, head injury, and seizures. These can be excluded by appropriate investigations such as computed tomography (CT) scan of the brain, lumbar puncture, and blood tests. The differential diagnosis of methanol toxicity is listed in Table 1.

The most characteristic MR findings in methanol toxicity are bilateral putaminal necroses with varying degrees of hemorrhage. This finding is by no means specific to methanol toxicity but is seen in a variety of conditions, including Wilson disease, Leigh disease, and hypoxic ischemic encephalopathy. Putaminal necrosis and hemorrhage result from the direct toxic effects of methanol metabolites and metabolic acidosis in the basal ganglia. Cerebral and intraventricular hemorrhage, cerebellar necrosis, diffuse cerebral edema, bilateral subcortical white matter necrosis or edema, and optic nerve necrosis have been described in severe methanol intoxication. ${ }^{[1]}$

Methanol toxicity is eminently treatable; however, untreated cases or delay in initiating treatment results in poor prognosis. In a recent study, poor prognosis was associated with $\mathrm{pH}<7$, coma on admission, and $>24 \mathrm{~h}$ delay from intake to admission. ${ }^{[12]}$ Methanol toxicity, if untreated, can have a high fatality rate. In three recent outbreaks of methyl alcohol poisoning, the case fatality rates were 10\% (2013, Libya), 29\% (May 2014, Kenya), and 21\% (July 2014, Kenya). ${ }^{[13]}$

The management of methanol poisoning includes standard supportive care, the correction of metabolic acidosis, the administration of folinic acid, the provision of an antidote (ethanol or fomepizole) to inhibit the metabolism of methanol to formate, and selective hemodialysis to correct severe metabolic abnormalities and to enhance methanol and formate elimination. ${ }^{[14]}$ Fomepizole is usually given as a bolus dose of $15 \mathrm{mg} / \mathrm{kg}$ intravenous diluted in isotonic saline,

\begin{tabular}{l}
\hline Table I: Differential diagnosis of methanol toxicity \\
\hline Ethylene glycol toxicity \\
Diethylene glycol toxicity \\
Propylene glycol toxicity \\
Alcoholic ketoacidosis \\
Carbon monoxide poisoning \\
Neuroleptic malignant syndrome \\
Viral encephalitis \\
Bacterial meningitis \\
Head injury \\
Seizures
\end{tabular}

followed by $10 \mathrm{mg} / \mathrm{kg}$ every $12 \mathrm{~h}$ (every $4 \mathrm{~h}$ during hemodialysis); ethanol is administered both intravenously as a $10 \%$ solution in $5 \%$ glucose and per oral in boluses of $20 \%$ solution. ${ }^{[2]}$ Both fomepizole and ethanol have equal efficacy in the treatment of methanol toxicity. ${ }^{[2]}$ However, the adverse events are more commonly seen with ethanol as compared to fomepizole. In a large series, adverse events were seen in $20 \%$ of ethanol-treated patients as compared to $5 \%$ in fomepizole-treated patients. ${ }^{[15]}$ Similarly, serious adverse events were seen in $8 \%$ of ethanol-treated and $2 \%$ of fomepizole-treated patients. Common adverse events noted with ethanol treatment were coma, severe agitation, respiratory depression, and hypotension. Coma, hypotension, and bradycardia were the common adverse events noted with fomepizole treatment. In terms of availability, however, ethanol scores over fomepizole as ethanol is widely available. Fomepizole is also most costly than ethanol.

The American Academy of Clinical Toxicology has published practice guidelines, which are very helpful in managing patients with methanol toxicity ${ }^{[14]}$ The salient points in the guidelines include as follows:

- For the patients presenting with ophthalmologic abnormalities or significant metabolic acidosis, the acidosis should be corrected with intravenous sodium bicarbonate

- Further generation of toxic metabolite, formic acid should be blocked by the administration of ethanol or fomepizole

- Formic acid metabolism should be enhanced by the administration of intravenous folinic acid

- Hemodialysis may be done in severe cases to correct severe metabolic abnormalities and enhance methanol and formate elimination

- Supportive care is aimed at airway management, correct of electrolyte abnormalities, and adequate hydration.

\section{Conclusion}

In a comatose patient, a combination of metabolic acidosis (high anion gap and high osmolar gap) and bilateral putaminal necrosis on CT/MRI is virtually diagnostic of methanol toxicity. Early diagnosis and prompt initiation of treatment can go a long way in ensuring a good outcome for the patient.

\section{Financial support and sponsorship Nil.}

\section{Conflicts of interest}

There are no conflicts of interest. 


\section{References}

1. Posner JB, Saper CB, Schiff ND, Plum F. Plum and Posner's Diagnosis of Stupor and Coma. ${ }^{\text {th }}$ ed. New York: Oxford University Press; 2007.

2. Zakharov S, Pelclova D, Navratil T, Belacek J, Komarc M, Eddleston M, et al. Fomepizole versus ethanol in the treatment of acute methanol poisoning: Comparison of clinical effectiveness in a mass poisoning outbreak. Clin Toxicol (Phila) 2015;53:797-806.

3. Brahmi N, Blel Y, Abidi N, Kouraichi N, Thabet H, Hedhili A, et al. Methanol poisoning in Tunisia: Report of 16 cases. Clin Toxicol (Phila) 2007;45:717-20

4. Anderson TJ, Shuaib A, Becker WJ. Methanol poisoning: Factors associated with neurologic complications. Can J Neurol Sci $1989 ; 16: 432-5$.

5. Ley CO, Gali FG. Parkinsonian syndrome after methanol intoxication. Eur Neurol 1983;22:405-9.

6. Sebe A, Satar S, Uzun B, Topal M, Yesilagac H, Avci A. Intracranial hemorrhage associated with methanol intoxication. Mt Sinai J Med 2006;73:1120-2.

7. Bansal H, Bansal A, Kachhap MN, Chowdhary A, Koka PS. Therapeutic Application of bone marrow-derived stem cells in a patient with methanol-induced blindness. J Stem Cells 2015;10:1-11.

8. Khan AH, Rahaman MF, Mollah RI, Alam A, Hassan SN, Chowdhury MA. Methanol induced toxic amblyopia - A case report.
Mymensingh Med J 2016;25:176-8,

9. Hovda KE, Hunderi OH, Rudberg N, Froyshov S, Jacobsen D. Anion and osmolal gaps in the diagnosis of methanol poisoning: Clinical study in 28 patients. Intensive Care Med 2004;30:1842-6.

10. Kraut JA, Kurtz I. Toxic alcohol ingestions: Clinical features, diagnosis, and management. Clin J Am Soc Nephrol 2008;3:208-25.

11. Blaneo M, Casado R, Vázquez F, Pumar JM. CT and MR imaging findings in methanol intoxication. AJNR Am J Neuroradiol 2006;27:452-4.

12. Hassanian-Moghaddam H, Pajoumand A, Dadgar SM, Shadnia SH Prognostic factors in methanol poisoning. Hum Exp Toxicol 2007;26:583-6.

13. Rostrup M, Edwards JK, Abukalish M, Ezzabi M, Some D, Ritter H, et al. The Methanol poisoning outbreaks in libya 2013 and Kenya 2014. PLoS One 2016;11:e0157256.

14. Barceloux DG, Bond GR, Krenzelok EP, Cooper H, Vale JA; American Academy of Clinical Toxicology Ad Hoc Committee on the Treatment Guidelines for Methanol Poisoning. American Academy of Clinical Toxicology practice guidelines on the treatment of methanol poisoning. J Toxicol Clin Toxicol 2002;40:415-46.

15. Lepik KJ, Levy AR, Sobolev BG, Purssell RA, DeWitt CR, Erhardt GD, et al. Adverse drug events associated with the antidotes for methanol and ethylene glycol poisoning: A comparison of ethanol and fomepizole. Ann Emerg Med 2009;53:439-50. 